\title{
Evaluation of the efficacy of commercial protective cultures against mold and yeast in queso fresco
}

\author{
Ghadeer M. Makki, () Sarah M. Kozak, (1) Katharine G. Jencarelli, (๑) and Samuel D. Alcaine* (1) \\ Department of Food Science, Cornell University, Ithaca, NY 14853
}

\begin{abstract}
In this study, we evaluated the efficacy of 3 commercial protective cultures designated PC1 (Lactobacillus spp.), PC2 (Lactobacillus rhamnosus), and PC3 (Lactobacillus rhamnosus) as biopreservatives in queso fresco (QF) against 9 yeast strains (Candida zeylanoides, Clavispora lusitaniae, Debaryomyces hansenii, Debaryomyces prosopidis, Kluyveromyces marxianus, Meyerozyma guilliermondii, Pichia fermentans, Rhodotorula mucilaginosa, and Torulaspora delbrueckii) and 11 mold strains (Aspergillus cibarius, Aureobasidium pullulans, Penicillium chrysogenum, Penicillium citrinum, Penicillium commune, Penicillium decumbens, Penicillium roqueforti, Mucor genevensis, Mucor racemosus, Phoma dimorpha, and Trichoderma amazonicum). All fungal spoilage strains were previously isolated from dairy processing environments. A positive control (C) with no protective culture was included. Fungal spoilage organisms were inoculated on cheese surfaces at an inoculum level of $20 \mathrm{cfu} / \mathrm{g}$, and cheeses were stored at $6 \pm 2^{\circ} \mathrm{C}$ throughout the study. For yeast enumeration, cheeses were sampled on d $0,7,14$, and 21 postinoculation. Significant inhibition was detected for each yeast strain by comparing yeast counts for each cheese treated with protective culture against the control cheese using one-way ANOVA with Bonferroni correction performed individually at $\mathrm{d} 7,14$, and 21 postinoculation. Mold growth was visually observed and imaged weekly through $70 \mathrm{~d}$ postinoculation. Whereas PC3 inhibited Cl. lusitaniae, Mey. guilliermondii, and Ph. dimorpha, PC2 inhibited the outgrowth of Cl. lusitaniae, D. hansenii, and Ph. dimorpha. Protective culture 1 had the broadest spectrum of efficacy across yeast and molds, delaying spoilage caused by 4 distinct yeast strains $(\mathrm{Cl}$. lusitaniae, D. hansenii, D. prosopidis, and Mey. guil-
\end{abstract}

Received April 22, 2020

Accepted June 22, 2020

*Corresponding author: alcaine@cornell.edu liermondii), and inhibiting visible growth of 2 mold strains ( $P$. chrysogenum and $P h$. dimorpha). Results demonstrated that commercial protective cultures vary in performance, as indicated by the breadth of mold and yeast inhibition at both the genus and species level. This study suggests that manufacturers looking into using protective cultures should investigate their efficacy against specific fungal strains of concern.

Key words: queso fresco, protective culture, mold and yeast, biopreservative

\section{INTRODUCTION}

The popularity of fresh Hispanic-style cheeses is increasing in the United States, given the growing Hispanic population, which is forecasted to reach 93.8 million by 2045 (United State Census Bureau, 2018). Queso fresco ( $\mathbf{Q F}$ ) is regarded as the most popular fresh Hispanic-style cheese in the United States (TorresLlanez et al., 2006; Van Hekken et al., 2017). Having no federal standard of identity has resulted in a wide range of variation in QF composition (Brown et al., 2018). However, the high moisture content (46-57\%) and $\mathrm{pH}$ value $(>5.8)$, together with relatively low salt levels $(1-3 \%)$ of $\mathrm{QF}$, provide a favorable medium for the growth of spoilage organisms and limits the shelf life to 8 wk under refrigeration (Guo et al., 2011; Van Hekken et al., 2017).

On average, 5 to $10 \%$ of food is lost annually due to fungal spoilage, jeopardizing food safety and threatening sustainability (Pitt and Hocking, 2009; Garnier et al., 2017). Fungal spoilage negatively affects the consumer experience by creating off-flavors and changing the texture of food. Furthermore, the ability of some molds to produce mycotoxins can endanger consumer health (Fernandez et al., 2017; Garnier et al., 2017). Hurdle technologies have been leveraged to decrease the incidence of spoilage in fresh cheese, including milk pasteurization, use of preservatives, and modifiedatmosphere packaging. However, postpasteurization contamination remains an issue of concern, and fungal resistance to traditional preservatives adds to the chal- 
lenges facing the industry. For example, some Penicillium spp. have developed the ability to reduce sorbates to 4-hexanol or 4-hexanoic acid, compromising sorbate antifungal activity and creating off-flavors (Pitt and Hocking, 2009; Fernandez et al., 2017). In addition to these challenges, consumers concerned about the ingredients in foods are increasingly demanding "clean label" products. This puts pressure on manufacturers and researchers to exploit naturally occurring alternatives to enhance food safety and quality while keeping up with consumer trends for clean labels (Fernandez et al., 2017). Biopreservation is gaining traction as a means to leverage food safety and potentially extend shelf life using naturally occurring agents (Cheong et al., 2014; Galvez et al., 2014).

Biopreservation utilizes the natural microbiota or its microbial constituents to maintain quality and safety in foods (Stiles, 1996). Lactic acid bacteria (LAB) are non-spore-forming, anaerobic or facultative aerobic gram-positive bacteria (Ben Said et al., 2019). Given the GRAS (generally regarded as safe by the United States Food and Drug Administration) status of LAB and their long history of use in fermentation, there is growing interest in exploiting their potential to function as bioprotective cultures (Crowley et al., 2013). The LAB have been shown to exhibit antifungal activity, the mechanisms of which include (1) secreting organic acids such as lactic, phenyllactic, propionic, and acetic acids that render the medium unsuitable for the growth of some microorganisms (Cheong et al., 2014; Galvez et al., 2014); (2) producing metabolites that interfere with DNA synthesis, such as reuterin produced by Lactobacillus reuteri (Galvez et al., 2014); and (3) secreting fatty acids, dipeptides, bacteriocins, and other proteinaceous compounds with antifungal activity (Crowley et al., 2013).

Several commercially available LAB bioprotective cultures are currently marketed for their antifungal efficacy, but there is limited published data by which to gauge how these cultures compare in the spectrum of antifungal activity and their potential for shelf-life improvement in QF. This study was carried out to evaluate the efficacy of 3 commercially available LAB cultures advertised as providing protection against fungal spoilage in fresh cheese, and provide a benchmark for QF manufacturers on which cultures may be most effective for their products. We hypothesized that antifungal efficacy would vary between different LAB cultures depending upon both genus and species of the challenge organisms; thus, one protective culture may be more ideally suited to protect QF against a specific spoilage organism.

\section{MATERIALS AND METHODS}

\section{Protective Culture Selection and Characterization}

Three commercial protective cultures designated PC1 (Lactobacillus spp.), PC2 (L. rhamnosus), and PC3 (L. rhamnosus) were investigated throughout the study to evaluate their efficacy against fungal spoilage organisms in QF. Protective cultures were sourced from 3 manufacturers that are not disclosed here for proprietary reasons. All protective cultures were stored frozen at -80 $\pm 2^{\circ} \mathrm{C}$ until use. Protective cultures were resuspended in PBS and plated on de Man, Rogosa, and Sharpe agar (Hardy Diagnostics, Santa Maria, CA). Plates were incubated at $30^{\circ} \mathrm{C}$ for $48 \mathrm{~h}$, aerobically. Five colonies of each protective culture treatment were selected for 16s rDNA sequencing using16S-PEU7-Forward Primer (5'-GCAAACAGGATTAGATACCC-3') and the 16SDG74-Reverse Primer (5'-AGGAGGTGATCCAACCGCA-3'). Overnight cultures of each individual colony were grown in MRS broth to make enzymatic lysates. After amplification, PCR products were cleaned up using exonuclease I and shrimp alkaline phosphatase, and sent to the Biotechnology Resource Center at Cornell University (Ithaca, NY) for sequencing. Sequence analysis was performed using Geneious version 11.1.5 (Auckland, New Zealand).

\section{Fungal Spoilage Organisms}

Yeast and mold strains used for this study were sourced from the Cornell University Food Safety Laboratory and the Alcaine Research Group (Cornell University, Ithaca, NY). Nine yeast strains (Candida zeylanoides, Clavispora lusitaniae, Debaryomyces hansenii, Debaryomyces prosopidis, Kluyveromyces marxianus, Meyerozyma guilliermondii, Pichia fermentans, Rhodotorula mucilaginosa, and Torulaspora delbrueckii) were used throughout the study. Eleven species of mold spanning 5 genera were included in the study (Aspergillus cibarius, Aureobasidium pullulans, Penicillium chrysogenum, Penicillium citrinum, Penicillium commune, Penicillium decumbens, Penicillium roqueforti, Mucor genevensis, Mucor racemosus, Phoma dimorpha, and Trichoderma amazonicum).

All strains were previously isolated from dairy processing environments by the Food Safety Laboratory of Cornell University, and stocks were stored frozen at $-80 \pm 2^{\circ} \mathrm{C}$. Table 1 lists the genus and species identification, organism classification, and isolation source of fungal spoilage organisms used in the study. More information regarding the isolated strains is available 
in the Food Microbe Tracker database (foodmicrobetracker.com).

\section{Preparation of Yeast Inoculum}

To prepare yeast inocula, frozen yeast isolates were streaked onto potato dextrose agar (PDA) plates (Hardy Diagnostics) followed by incubation at $25^{\circ} \mathrm{C}$ for 48 h. From each plate, a single colony was selected with a sterile loop to inoculate $5 \mathrm{~mL}$ of potato dextrose broth (HiMedia Laboratories Pvt. Ltd., Mumbai, India) followed by incubation at $25^{\circ} \mathrm{C}$ for $16 \mathrm{~h}$. Cultures were then vortexed with glycerol at $50 \%$ (vol/vol) to form yeast stocks. Stocks were stored at $-80 \pm 2^{\circ} \mathrm{C}$ until use. To enumerate yeast strain stocks, the stocks were serially diluted in PBS and plated on PDA, followed by incubation at $25^{\circ} \mathrm{C}$ for $48 \mathrm{~h}$. Yeast colony counts were counted using a Color Q-Count (Advanced Instruments, Norwood, MA).

\section{Preparation of Mold Spore Suspensions}

To prepare mold spore suspensions, frozen mold isolates were spotted onto malt extract agar plates (Difco, BD Diagnostics, Franklin Lakes, NJ) followed by incubation at $25^{\circ} \mathrm{C}$ for $30 \mathrm{~d}$ to allow for spore formation. Each plate was flooded with PBS containing 0.1\% tween 80 (Tokyo Chemical Industry Co. Ltd., Tokyo, Japan). Plate surfaces were gently scraped using sterile cell spreaders to free spores and mycelia. To remove mycelia, the mold suspensions were filtered through 4 layers of sterile cheese cloth. Spore formation was confirmed microscopically (Reichert Technologies, Depew, NY). In sterile 50-mL Falcon tubes (VWR, Radnor, PA), spore suspensions were mixed with glycerol at $50 \%$ ( vol/vol) for storage. Spore suspensions were stored at $-80 \pm 2{ }^{\circ} \mathrm{C}$ until use.

For each mold strain, the concentrations of spore suspensions were determined microscopically using a Neubauer's improved counting chamber hemocytometer (Paul Marienfeld GmbH \& Co. KG, LaudaKönigshofen, Germany). Spore counts were confirmed by plating in duplicate on dichloran rose bengal chloramphenicol agar (Becton, Dickinson and Co., Sparks, $\mathrm{MD}$ ) followed by incubation at $25^{\circ} \mathrm{C}$ for $5 \mathrm{~d}$.

\section{Cheese Making and Inoculation with Protective Cultures}

Immediately before cheese making, each commercial protective culture was resuspended in pasteurized, homogenized whole milk sourced from the Cornell dairy plant (Ithaca, NY).

Queso fresco was made on a bench-top scale following the method in Guo et al. (2011) and Leggett et al. (2012) with a few modifications (Lawton et al., 2020). Pasteurized homogenized whole milk that was previously standardized was sourced from the Cornell dairy plant and warmed to $32^{\circ} \mathrm{C}$. At this point, coagulating agents were added, consisting of $0.1 \%$ (wt/vol) calcium

Table 1. Genus and species identification, type, location, and isolation source for spoilage organisms

\begin{tabular}{llll}
\hline Strain $^{1}$ & Type & ID $^{2}$ & Isolate source \\
\hline Candida zeylanoides & Yeast & B90031 & Cheese \\
Clavispora lusitaniae & Yeast & B90007 & Raw milk \\
Debaryomyces hansenii & Yeast & B90013 & Cheese \\
Debaryomyces prosopidis & Yeast & B90028 & Cheese \\
Kluyveromyces marxianus & Yeast & B90008 & Raw milk \\
Meyerozyma guilliermondii & Yeast & E20377 & Yogurt \\
Pichia fermentans & Yeast & B90001 & Raw milk \\
Rhodotorula mucilaginosa & Yeast & E20331 & Dairy processing environment \\
Torulaspora delbrueckii & Yeast & E20442 & Yogurt \\
Aspergillus cibarius & Mold & E20323 & Dairy processing environment \\
Aureobasidium pullulans & Mold & E20290 & Yogurt \\
Mucor genevensis & Mold & TD0021 & Yogurt \\
Mucor racemosus & Mold & E20368 & Yogurt \\
Penicillium chrysogenum & Mold & E20332 & Dairy processing environment \\
Penicillium citrinum & Mold & E20297 & Yogurt \\
Penicillium commune & Mold & B90026 & Cheese \\
Penicillium decumbens & Mold & E20320 & Dairy processing environment \\
Penicillium roqueforti & Mold & E20329 & Dairy processing environment \\
Phoma dimorpha & Mold & E20369 & Yogurt \\
Trichoderma amazonicum & Mold & E20387 & Yogurt \\
\hline
\end{tabular}

${ }^{1}$ All strains were from the Food Safety Laboratory isolate collection at Cornell, except for Mucor genevensis, which was from the Alcaine Research Group isolate collection (Cornell University, Ithaca, NY).

${ }^{2}$ ID refers to identification in Food Microbe Tracker database (www.foodmicrobetracker.com). 
chloride (GetCulture Inc., Madison, WI) and chymosin (Chy-Max Extra 73812, Chr. Hansen Inc., Milwaukee, WI) at a rate of $130 \mathrm{~mL} / 1,000 \mathrm{~L}$ of milk. For each treatment, the respective protective culture was inoculated at the manufacturer's recommended dosage rate. A control cheese $(\mathbf{C})$, without addition of protective culture (PC), was included in the study. After $45 \mathrm{~min}$ of incubation at $32^{\circ} \mathrm{C}$, curds were cut and allowed to heal for $10 \mathrm{~min}$. The curds were cooked by increasing the temperature gradually over $30 \mathrm{~min}$ until curd temperature reached $39^{\circ} \mathrm{C}$. Curds were then cooked at $39^{\circ} \mathrm{C}$ for an additional $30 \mathrm{~min}$. Whey, at a volume equivalent to $10 \%$ of the initial milk volume, was drained and a brine solution of $0.16 \mathrm{~g} / \mathrm{mL}$ sodium chloride (Avantor Performance Materials LLC, Radnor, PA) was added and incubated for $20 \mathrm{~min}$. Curds were transferred to sterile cheese cloth and allowed to drain for $1 \mathrm{~h}$.

For the yeast challenge study, drained curd was added to 6 -well plates $(3.5 \mathrm{~cm}$ in diameter, Falcon, Corning, NY), which were used as cheese molds. To each well, $13 \pm 0.2 \mathrm{~g}$ of curd was aseptically added. Cheeses were pressed for $16 \mathrm{~h}$ at room temperature with 4.5-lb weights, with cheeses having a final weight of $\sim 5 \mathrm{~g}$.

For the mold challenge study, drained curd was added to 12 -well plates $(2.2 \mathrm{~cm}$ in diameter, Falcon), which were used as cheese molds. To each well, $6 \pm 0.1$ $\mathrm{g}$ of curd was aseptically added. Cheeses were pressed for $16 \mathrm{~h}$ at room temperature with 4.5 -lb weights. The final weight of individual cheeses after pressing was $\sim 4$ g. Figure 1 shows a diagram of the QF cheesemake procedure.

Moisture and $\mathrm{pH}$ values were measured for 2 samples per treatment using a CEM microwave oven (CEM Corp., Matthews, NC) and SevenGo pH meter (Mettler Toledo, Columbus, OH), respectively.

\section{Yeast Challenge Study}

For each yeast strain, four 6-well plates were prepared and a single plate was assigned to each treatment (C, PC1, PC2, and PC3). Yeast strains were inoculated on the surface of cheese samples with a target inoculum of $20 \mathrm{cfu} / \mathrm{g}$. Throughout the study, plates were stored under refrigeration $\left(6 \pm 2^{\circ} \mathrm{C}\right)$.

To enumerate yeast levels at $\mathrm{d} 0,7,14$, and 21 postinoculation, the contents of one well were aseptically transferred to a sterile stomacher bag (Whirl-Pak, Nasco, Fort Atkinson, WI) where $45 \mathrm{~mL}$ of PBS was added to achieve a 1:9 ratio followed by homogenization at $230 \mathrm{rpm}$ for $60 \mathrm{~s}$ using a Seward Stomacher 400 Circulator blender (Seward Ltd., Worthing, UK). Samples were serially diluted and plated in duplicate on PDA supplemented with $25 \mathrm{mg}$ of chloramphenicol per liter (Sigma-Aldrich Corp., St. Louis, MO). Plates
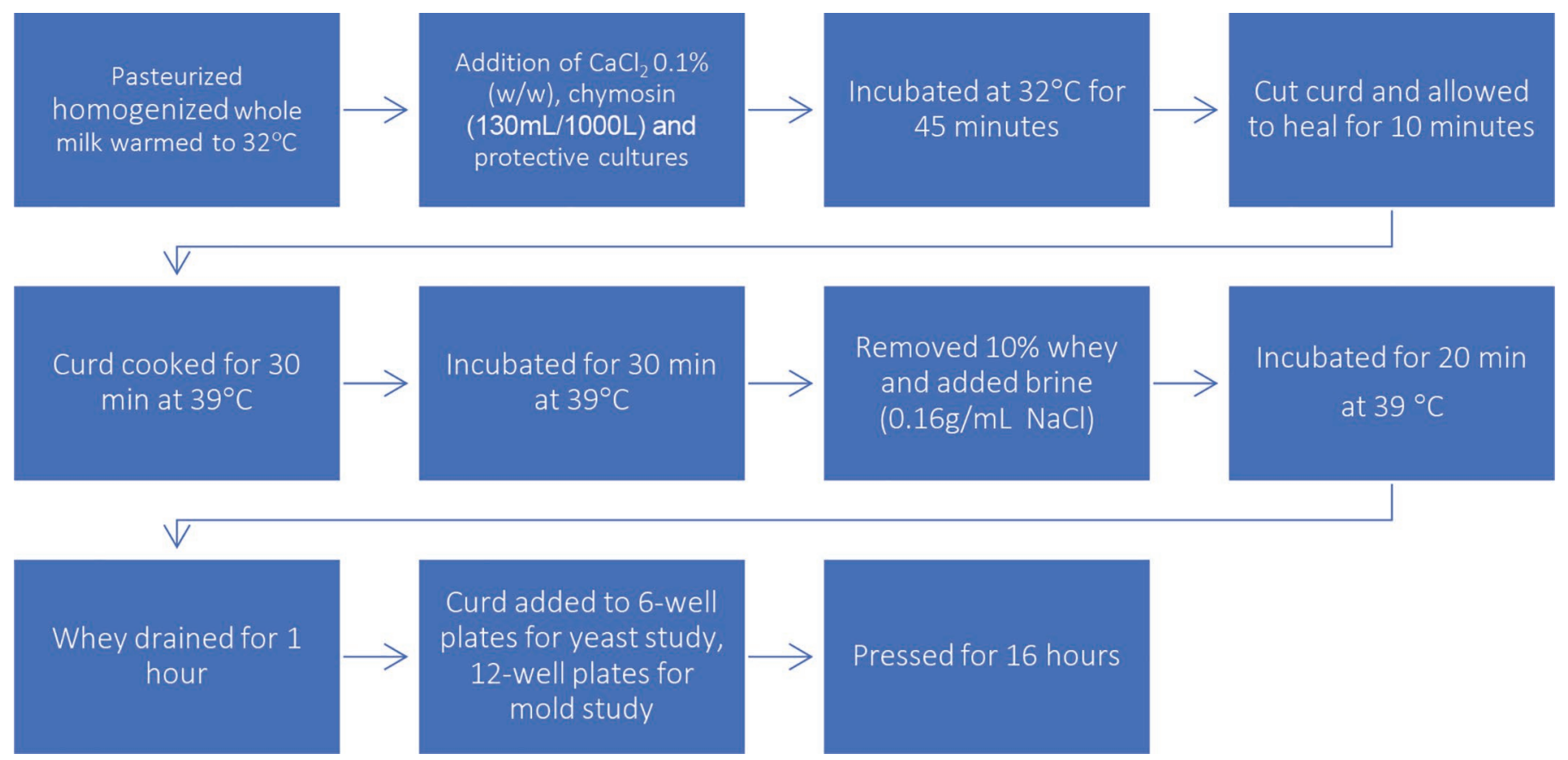

Figure 1. Queso fresco make process modified from that described in Lawton et al. (2020). Protective cultures were added at the manufacturer's suggested dosage rate. 

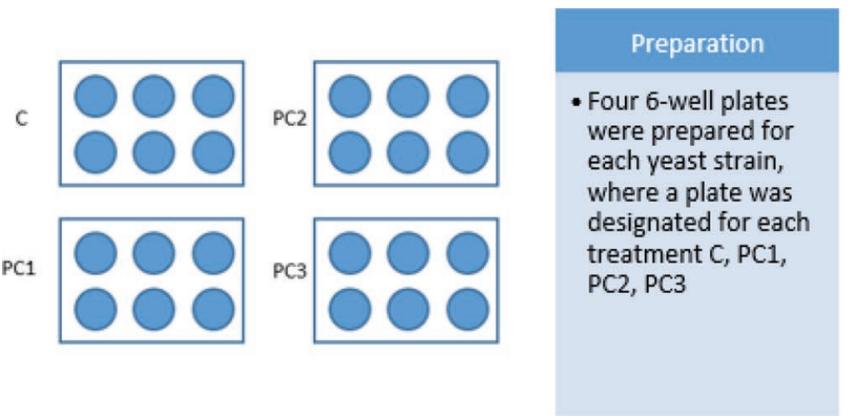
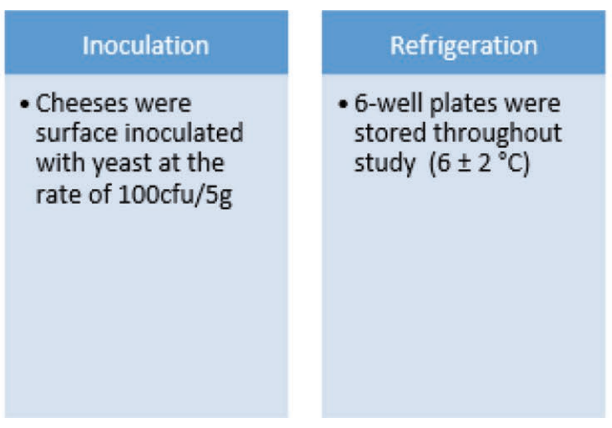

Figure 2. Schematic for preparing 6-well plates for yeast challenge study, where C is control with no protective culture, and PC1, PC2, and PC3 are protective cultures of Lactobacillus spp., Lactobacillus rhamnosus, and L. rhamnosus, respectively. Samples were stored refrigerated at $6 \pm 2^{\circ} \mathrm{C}$ throughout the study.

were incubated at $25^{\circ} \mathrm{C}$ for $5 \mathrm{~d}$. Figure 2 shows a schematic outlining the experimental design for the yeast challenge study.

\section{Mold Challenge Study}

Following QF pressing, a single 12-well plate was prepared for each challenged mold strain where C, PC1, PC2, and PC3 represent the first, second, third, and fourth columns of wells, respectively, of the plate (Figure 3). A negative control plate was included in the study, in which treatments were not inoculated with mold. Mold strains were inoculated on the surface of cheese samples in each well at a target inoculum of 25 $\mathrm{cfu} / \mathrm{g}$. Plates were stored at refrigeration temperature $\left(6 \pm 2^{\circ} \mathrm{C}\right)$ throughout the study.

To assess mold outgrowth on QF, plates were visually observed at d $0,7,14,21,28,35,42,49,56$, 63 , and 70 postinoculation and imaged using Canon PowerShot SX530 160Megapixel HS Digital Camera (Canon, Tokyo, Japan). We chose $70 \mathrm{~d}$ as the duration of this study because the shelf life of QF is relatively short, and the QF used in this study started to show mold growth on the negative controls beyond $70 \mathrm{~d}$. Furthermore, most molds did not grow until at least $14 \mathrm{~d}$ of storage. The following scale was used to report results: $-=$ no visible mold growth in all replicates, $+^{*}$ $=$ cheeses transitioning into matte appearance in some replicates, $+=$ matte appearance with no colored mold growth in all replicates, $++^{*}=$ cheeses transitioning into colored mold growth in some replicates, and $++=$ mold growth with color change in all replicates. A schematic for the design of mold challenge study is shown in Figure 3.

\section{Statistical Analysis}

Experiments were performed in triplicate for both yeast and mold studies. Significant $(P<0.05)$ inhibition of individual yeast strains was determined by comparing average yeast counts $(\log \mathrm{cfu} / \mathrm{g})$ for each PC treatment against the control cheese with no protec-
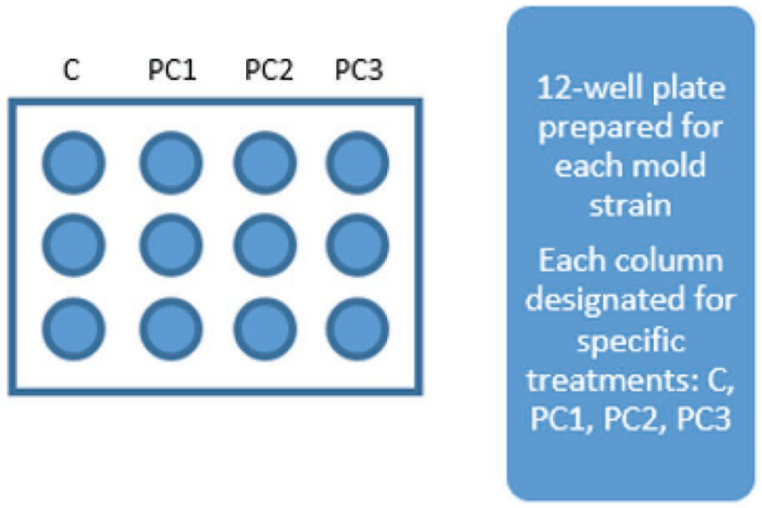
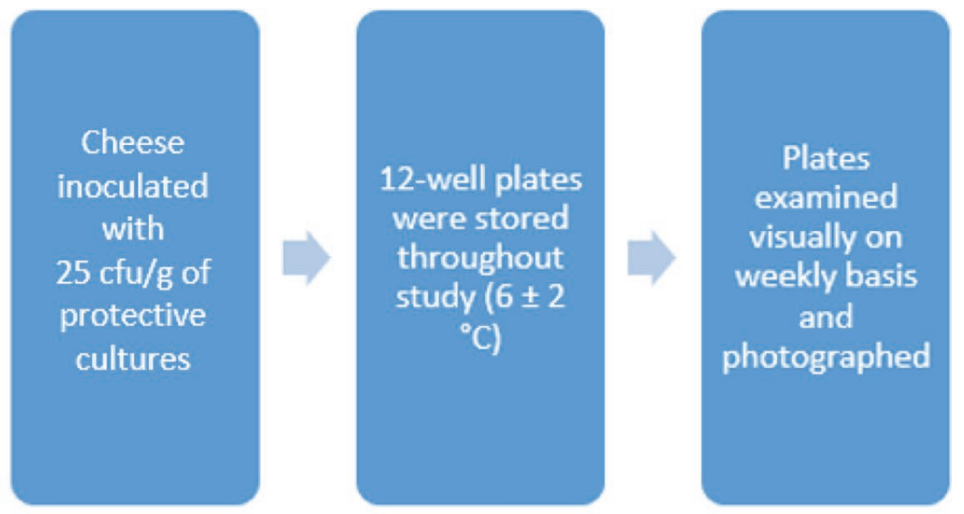

Figure 3. Schematic of mold challenge study on queso fresco where cheeses were surface inoculated at a target level of $25 \mathrm{cfu} / \mathrm{g}$. Treatments: $\mathrm{C}$ is control with no protective culture, and PC1, PC2, and PC3 are protective cultures of Lactobacillus spp., Lactobacillus rhamnosus, and L. rhamnosus, respectively. All 12-well plates were stored refrigerated at $6 \pm 2^{\circ} \mathrm{C}$ throughout the study. 
tive culture using a one-way ANOVA with Bonferroni correction performed individually at d 7, 14, and 21 postinoculation. For this study, a biological difference was defined as having both a significant difference $(P$ $<0.05$ ) and at least a 1-log difference between the treatment and the control. Statistical analysis was performed using JMP Pro software version 14 (SAS Institute Inc., Cary, NC).

\section{RESULTS AND DISCUSSION}

In this study, laboratory-scale QF cheeses were made individually to challenge the growth of mold and yeast strains through biopreservation with commercially available LAB cultures. The species of each protective culture was determined by $16 \mathrm{~S}$ rDNA sequencing. For $\mathrm{PC} 1$, we confirmed that the culture contained Lactobacillus spp., with 4 of the 5 colonies identified as Lactobacillus plantarum and the other as Lactobacillus rhamnosus/paracasei. All colonies for PC2 and PC3 genotypically matched Lactobacillus rhamnosus/ paracasei. Growth of protective cultures throughout the study was not determined because of the inability to distinguish between native $\mathrm{LAB}$ and added protective cultures.

Although there is no federal standard of identity for $\mathrm{QF}$, the moisture and $\mathrm{pH}$ measurements indicated that the laboratory-scale cheese model simulated the characteristics of traditionally made QF. For the yeast challenge study, the average moisture values were 61.89 $\pm 1.82,60.76 \pm 2.23,60.28 \pm 1.59$, and $60.95 \pm 1.42 \%$ and average $\mathrm{pH}$ values were $6.42 \pm 0.06,5.99 \pm 0.07$, $6.30 \pm 0.05$, and $6.34 \pm 0.03$ for $\mathrm{C}, \mathrm{PC} 1, \mathrm{PC} 2$, and PC3, respectively. For the mold challenge study, the average moisture results were $61.88 \pm 1.79,59.76 \pm$ $3.08,60.38 \pm 2.15$, and $59.82 \pm 0.98 \%$ and average $\mathrm{pH}$ values were $6.50 \pm 0.11,6.07 \pm 0.17,6.37 \pm 0.08$, and $6.40 \pm 0.06$ for $\mathrm{C}, \mathrm{PC} 1, \mathrm{PC} 2$, and $\mathrm{PC} 3$, respectively. These results indicate that although moisture content remained consistent across treatments, lowering of the $\mathrm{pH}$ compared with control indicated the expected acidification by the protective cultures.

Yeast studies were carried out for $21 \mathrm{~d}$, based on the relatively short shelf life of QF (Bermúdez-Aguirre and Barbosa-Cánovas, 2010). Yeast counts below the detection limit were assigned a value of $1.3 \mathrm{log} \mathrm{cfu} / \mathrm{g}$. In the context of this study, statistical significance was detected at $P<0.05$, whereas a difference in biological meaning was determined at both $P<0.05$ and at difference of at least $1 \log \mathrm{cfu} / \mathrm{g}$ compared with the control cheese without a protective culture. Based on the literature, quality characteristics of food products deteriorate as yeast counts exceed $5 \mathrm{log} \mathrm{cfu} / \mathrm{g}$. Beyond that level, textural changes, off-flavors, and visual defects become detectable (Zantar et al., 2014). Therefore, a successful treatment will keep yeast counts below this level throughout the entire sampling period, or extend the shelf life by keeping yeast counts below the spoilage limit longer than in cheese without protective culture.

The efficacy of the protective cultures against the selected yeast challenge strains varied widely. For some yeast strains, protective cultures were not effective at preventing yeast counts from reaching the spoilage limit of $5 \mathrm{log} \mathrm{cfu} / \mathrm{g}$ throughout the entirety of $21 \mathrm{~d}$ of storage. For $C$. zeylanoides, the spoilage limit was exceeded by d 7 for all cheeses except for PC1-treated cheese, in which the spoilage limit was reached between $\mathrm{d} 7$ and 14, highlighting the ability of PC1 to delay spoilage (Figure 4A). At d 21, no significant difference was observed for C. zeylanoides counts in the C, PC1, PC2, and PC3 treatments $(P>0.05)$. Similarly, D. prosopidis counts reached $5 \mathrm{log} \mathrm{cfu} / \mathrm{g}$ across all treatments by d 14 (Figure 4D). At this time, a biological difference was observed between D. prosopidis counts on PC1- and $\mathrm{PC} 2$-treated cheeses and $\mathrm{C}$ cheese, with average counts of $5.16 \pm 1.67$ and $5.75 \pm 1.21 \mathrm{cfu} / \mathrm{g}$ on PC1 and PC2, respectively, and $8.02 \pm 0.59 \log \mathrm{cfu} / \mathrm{g}$ on $\mathrm{C}(P<0.05)$. Although protective cultures managed to delay yeast growth over $21 \mathrm{~d}$ compared with control, the spoilage limit was reached across treatments by $14 \mathrm{~d}$ of storage. Similarly, PC1, PC2, and PC3 delayed the outgrowth of $R$. mucilaginosa until d 14 . At d 14, cheeses inoculated with protective cultures had significantly lower counts of $R$. mucilaginosa compared with positive control cheese $(P=0.0018, P=0.0009$, and $P=0.0018$, respectively, Figure $4 \mathrm{H})$. The $R$. mucilaginosa counts on PC1-, PC2-, and PC3-inoculated cheeses were below the spoilage limit, compared with $\mathrm{C}$, which was above the spoilage limit (Table 2). However, at d 21, although cheeses inoculated with protective cultures continued to have significantly lower counts of $R$. mucilaginosa $(P=0.000351, P<0.0001$, and $P<0.0001$, respectively) compared with $\mathrm{C}$, all were above the spoilage limit. These data suggest the potential efficacy of PC1, $\mathrm{PC} 2$, and $\mathrm{PC} 3$ in delaying QF spoilage caused by $R$. mucilaginosa under refrigerated conditions for less than $14 \mathrm{~d}$.

Some yeast strains reached the spoilage limit of $5 \log$ $\mathrm{cfu} / \mathrm{g}$ by $21 \mathrm{~d}$ in cheeses without the addition of protective cultures, whereas cheeses with protective cultures either reached the spoilage limit later or counts did not reach the spoilage limit throughout the storage period. Although the growth of $C l$. lusitaniae, D. hansenii, $K$. marxianus, and Mey. guilliermondii reached the spoilage limit in the $\mathrm{C}$ cheeses, growth of these strains was inhibited by one or more of the protective cultures, 
preventing them from reaching the spoilage limit. For Cl. lusitaniae, counts at d 14 in PC1-, PC2-, and PC3inoculated cheeses were significantly lower than those in the control cheese $(P=0.0117, P<0.0001$, and $P=$ 0.0054, respectively, Figure 4B, Table 2). At d 21, Cl. lusitaniae counts on $\mathrm{PC} 1$ and $\mathrm{PC} 2$ were significantly lower than counts on $\mathrm{C}$, which surpassed the spoilage limit $(P=0.0009$ and $P=0.0126$, respectively). The average biological difference of 2.36 and $1.83 \mathrm{log} \mathrm{cfu} / \mathrm{g}$ was evident at d 21 comparing $C$ l. lusitaniae counts on control cheese to PC1- and PC2-treated cheeses, respectively.

For D. hansenii, cheeses inoculated with PC1 and PC2 had significantly lower yeast counts than the control at d $14(P=0.0081$ and $P=0.0324$ respectively, Figure $4 \mathrm{C}$ ). At this time, D. hansenii counts on cheeses inoculated with $\mathrm{PC} 1$ and $\mathrm{PC} 2$ differed from counts in the control by $>3 \log \mathrm{cfu} / \mathrm{g}$ (Table 2). At d 21, differences were detected for $D$. hansenii counts on cheeses inoculated with PC1, PC2, and PC3 compared with C
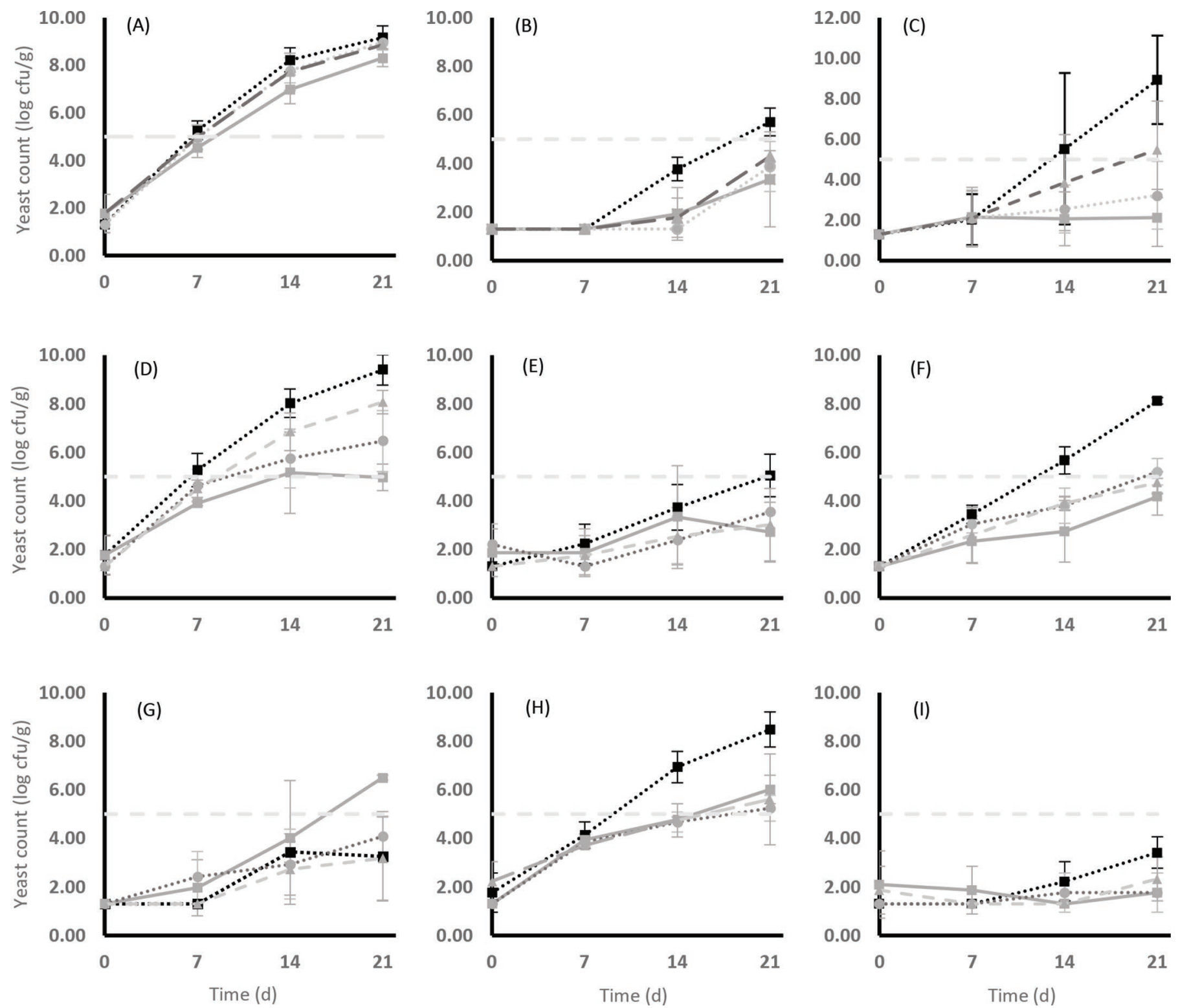

Figure 4. Growth of yeast strains on queso fresco at $6 \pm 2^{\circ} \mathrm{C}$ where $\mathrm{C}$ (black square) is control without protective culture, and $\mathrm{PC} 1$ (gray square), PC2 (gray circle), and PC3 (gray triangle) are different commercial protective cultures of Lactobacillus spp. (A) Candida zeylanoides, (B) Clavispora lusitaniae, (C) Debaryomyces hansenii, (D) Debaryomyces prosopidis, (E) Kluyveromyces marxianus, (F) Meyerozyma guilliermondii, (G) Pichia fermentans, (H) Rhodotorula mucilaginosa, and (I) Torulaspora delbrueckii. For counts that were below the detection limit, a value of $1.3 \mathrm{log} \mathrm{cfu} / \mathrm{g}$ was used. Error bars represent SD. The horizontal dashed line represents the cfu/g threshold for spoilage. 
$(P<0.0001, P<0.0001$, and $P=0.0297$, respectively $)$. A biological difference was observed for the same point comparing D. hansenii counts on cheeses inoculated with $\mathrm{PC} 1, \mathrm{PC} 2$, and $\mathrm{PC} 3$ and those on $\mathrm{C}$, with average values of $2.11 \pm 1.41,3.23 \pm 1.67,5.50 \pm 2.39$, and 8.94 $\pm 2.18 \log \mathrm{cfu} / \mathrm{g}$, respectively. For $D$. hansenii, the control cheese reached the spoilage limit between $\mathrm{d} 7$ and 14 postinoculation, whereas cheese treated with PC3 reached the spoilage limit between $\mathrm{d} 14$ and 21 postinoculation. Cheeses treated with PC1 and PC2 did not reach the spoilage limit throughout the study. These findings suggest potential efficacy of PC1 and PC2 in protecting against spoilage caused by $D$. hansenii as well as potential efficacy of PC3 in delaying spoilage caused by $D$. hansenii by an average of 1 wk compared with cheese with no protective culture.

No significant differences were noted for $K$. marxianus counts on cheeses treated with any of the protective cultures compared with control cheese at d 7, 14 and
21 postinoculation $(P>0.05$, Figure $4 \mathrm{E})$. Nonetheless, it is notable that only the control cheese reached the spoilage limit by d 21; PC1-, PC2-, and PC3-treated cheeses had $K$. marxianus counts below spoilage limits.

For Mey. guilliermondii, cheeses inoculated with PC1, PC2, and PC3 had significantly lower counts than the control cheese at d 14 and $21(P<0.05$ and $P<0.0001$, respectively, Figure $4 \mathrm{~F})$. At d 14, a biological difference was observed between Mey. guilliermondii counts on PC1-, PC2-, and PC3-inoculated cheeses and C cheese, with counts reaching the spoilage limit only in $\mathrm{C}$ (Table 4). By d 21, counts in the control reached $8.12 \pm 0.13$ $\log \mathrm{cfu} / \mathrm{g}$, whereas counts in treatment cheeses remained $\sim 3 \mathrm{log} \mathrm{cfu} / \mathrm{g}$ lower on average (Table 4). Our results indicated that $\mathrm{PC} 1, \mathrm{PC} 2$, and $\mathrm{PC} 3$ slowed growth of Mey. guilliermondii to different degrees. Cheese treated with PC1 and PC3 did not reach spoilage limits of Mey. guilliermondii through d 21 of refrigerated storage, whereas cheese treated with $\mathrm{PC} 2$ reached the spoilage

Table 2. Growth of yeast strains on queso fresco at $6 \pm 2{ }^{\circ} \mathrm{C}$ in different treatments

\begin{tabular}{|c|c|c|c|c|c|}
\hline Yeast & Treatment $^{1}$ & Day 0 & Day 7 & Day 14 & Day 21 \\
\hline \multirow[t]{4}{*}{ Candida zeylanoides } & $\mathrm{C}$ & $1.30 \pm 0.00$ & $5.27 \pm 0.39$ & $8.23 \pm 0.51$ & $9.17 \pm 0.49$ \\
\hline & PC1 & $1.77 \pm 0.81$ & $4.53 \pm 0.41$ & $6.99 \pm 0.60^{*}$ & $8.31 \pm 0.36$ \\
\hline & $\mathrm{PC} 2$ & $1.30 \pm 0.00$ & $4.97 \pm 0.54$ & $7.79 \pm 0.53$ & $8.97 \pm 0.32$ \\
\hline & PC3 & $1.77 \pm 0.81$ & $4.96 \pm 0.49$ & $7.73 \pm 0.78$ & $8.86 \pm 0.17$ \\
\hline \multirow[t]{4}{*}{ Clavispora lusitaniae } & $\mathrm{C}$ & $1.30 \pm 0.00$ & $1.30 \pm 0.00$ & $3.77 \pm 0.48$ & $5.71 \pm 0.57$ \\
\hline & PC1 & $1.30 \pm 0.00$ & $1.30 \pm 0.00$ & $1.93 \pm 1.09^{*}$ & $3.35 \pm 1.96^{*}$ \\
\hline & $\mathrm{PC} 2$ & $1.30 \pm 0.00$ & $1.30 \pm 0.00$ & $1.30 \pm 0.00^{*}$ & $3.88 \pm 1.03^{*}$ \\
\hline & PC3 & $1.30 \pm 0.00$ & $1.30 \pm 0.00$ & $1.77 \pm 0.81^{*}$ & $4.29 \pm 0.24$ \\
\hline \multirow[t]{4}{*}{ Debaromyces hansenii } & $\mathrm{C}$ & $1.30 \pm 0.00$ & $2.03 \pm 1.26$ & $5.53 \pm 3.74$ & $8.94 \pm 2.18$ \\
\hline & PC1 & $1.30 \pm 0.00$ & $2.15 \pm 1.47$ & $2.07 \pm 1.33^{*}$ & $2.11 \pm 1.41^{*}$ \\
\hline & $\mathrm{PC} 2$ & $1.30 \pm 0.00$ & $2.10 \pm 1.39$ & $2.55 \pm 1.18^{*}$ & $3.23 \pm 1.67^{*}$ \\
\hline & PC3 & $1.30 \pm 0.00$ & $2.08 \pm 1.36$ & $3.86 \pm 2.37$ & $5.50 \pm 2.39^{*}$ \\
\hline \multirow[t]{4}{*}{ Debaromyces prosopidis } & $\mathrm{C}$ & $1.77 \pm 0.81$ & $5.27 \pm 0.69$ & $8.02 \pm 0.59$ & $9.40 \pm 0.63$ \\
\hline & PC1 & $1.77 \pm 0.81$ & $3.90 \pm 0.10$ & $5.16 \pm 1.67^{*}$ & $4.97 \pm 0.55^{*}$ \\
\hline & $\mathrm{PC} 2$ & $1.30 \pm 0.00$ & $4.63 \pm 0.67$ & $5.75 \pm 1.21^{*}$ & $6.47 \pm 1.26^{*}$ \\
\hline & PC3 & $1.30 \pm 0.00$ & $4.50 \pm 0.36$ & $6.85 \pm 0.78$ & $8.07 \pm 0.48$ \\
\hline \multirow[t]{4}{*}{ Kluyveromyces marxianus } & $\mathrm{C}$ & $1.30 \pm 0.00$ & $2.23 \pm 0.81$ & $3.74 \pm 0.94$ & $5.05 \pm 0.88$ \\
\hline & PC1 & $1.87 \pm 0.98$ & $1.87 \pm 0.98$ & $3.34 \pm 2.12$ & $2.71 \pm 1.23$ \\
\hline & $\mathrm{PC} 2$ & $2.20 \pm 0.85$ & $1.30 \pm 0.00$ & $2.39 \pm 0.98$ & $3.54 \pm 0.00$ \\
\hline & PC3 & $1.30 \pm 0.00$ & $1.77 \pm 0.81$ & $2.55 \pm 1.18$ & $3.02 \pm 1.49$ \\
\hline \multirow[t]{4}{*}{ Meyerozyma guilliermondii } & $\mathrm{C}$ & $1.30 \pm 0.00$ & $3.45 \pm 0.37$ & $5.67 \pm 0.56$ & $8.12 \pm 0.13$ \\
\hline & PC1 & $1.30 \pm 0.00$ & $2.33 \pm 0.91$ & $2.75 \pm 1.27^{*}$ & $4.17 \pm 0.76^{*}$ \\
\hline & $\mathrm{PC} 2$ & $1.30 \pm 0.00$ & $3.03 \pm 0.35$ & $3.80 \pm 0.72^{*}$ & $5.20 \pm 0.55^{*}$ \\
\hline & PC3 & $1.30 \pm 0.00$ & $2.59 \pm 1.14$ & $3.90 \pm 0.28^{*}$ & $4.75 \pm 0.45^{*}$ \\
\hline \multirow[t]{4}{*}{ Pichia fermentans } & $\mathrm{C}$ & $1.30 \pm 0.00$ & $1.30 \pm 0.00$ & $3.44 \pm 0.43$ & $3.26 \pm 1.84$ \\
\hline & PC1 & $1.30 \pm 0.00$ & $1.97 \pm 1.15$ & $4.02 \pm 2.36$ & $6.50 \pm 0.11^{*}$ \\
\hline & $\mathrm{PC} 2$ & $1.30 \pm 0.00$ & $2.43 \pm 1.03$ & $2.94 \pm 1.44$ & $4.08 \pm 0.79$ \\
\hline & PC3 & $1.30 \pm 0.00$ & $1.30 \pm 0.00$ & $2.73 \pm 1.45$ & $3.18 \pm 1.73$ \\
\hline \multirow[t]{4}{*}{ Rhodotorula mucilaginosa } & $\mathrm{C}$ & $1.77 \pm 0.81$ & $4.13 \pm 0.54$ & $6.94 \pm 0.65$ & $8.48 \pm 0.72$ \\
\hline & PC1 & $1.30 \pm 0.00$ & $3.93 \pm 0.26$ & $4.77 \pm 0.11^{*}$ & $6.01 \pm 0.59^{*}$ \\
\hline & $\mathrm{PC} 2$ & $1.30 \pm 0.00$ & $3.85 \pm 0.22$ & $4.67 \pm 0.41^{*}$ & $5.24 \pm 0.53^{*}$ \\
\hline & PC3 & $2.23 \pm 0.81$ & $3.71 \pm 0.15$ & $4.74 \pm 0.68^{*}$ & $5.61 \pm 1.87^{*}$ \\
\hline \multirow[t]{4}{*}{ Torulaspora delbrueckii } & $\mathrm{C}$ & $1.30 \pm 0.00$ & $1.30 \pm 0.00$ & $2.23 \pm 0.81$ & $3.42 \pm 0.65$ \\
\hline & PC1 & $2.10 \pm 1.39$ & $1.87 \pm 0.98$ & $1.30 \pm 0.00$ & $1.77 \pm 0.81^{*}$ \\
\hline & $\mathrm{PC} 2$ & $1.30 \pm 0.00$ & $1.30 \pm 0.00$ & $1.77 \pm 0.81$ & $1.77 \pm 0.81^{*}$ \\
\hline & PC3 & $1.87 \pm 0.98$ & $1.30 \pm 0.00$ & $1.30 \pm 0.00$ & $2.33 \pm 0.91$ \\
\hline
\end{tabular}

${ }^{1} \mathrm{C}=$ control without protective culture; PC1, PC2, and PC3 = different commercial protective cultures of Lactobacillus spp.

${ }^{*} P<0.05$ : significant difference from control at a given time point. 
limit between d 14 and 21 of incubation under the same conditions. The results indicate the potential efficacy of $\mathrm{PC} 1$ and PC3 in protecting QF against spoilage caused by Mey. guilliermondii under refrigerated conditions.

For Pichia fermentans, no significant difference was detected at d 7 and 14 for yeast counts on cheese with protective cultures and control cheese (Figure 4G). However, at d 21 postinoculation, PC1-inoculated cheese had significantly higher counts than control cheese at the same time point, with counts being 3.24 $\log \mathrm{cfu} / \mathrm{g}$ higher on average $(P=0.0018)$. It is notable that only PC1-treated cheese reached the spoilage limit by $\mathrm{d} 21$ postinoculation, suggesting that, in some cases, protective cultures can enhance the growth of spoilage yeasts that are present, the opposite of the desired effect. The mechanism of action behind this phenomenon was not investigated in this study.

Torulaspora delbrueckii counts on QF at $\mathrm{d} 7$ and 14 postinoculation did not differ significantly $(P>0.05)$ among $\mathrm{PC} 1, \mathrm{PC} 2, \mathrm{PC} 3$, and $\mathrm{C}$ cheese at each respective time point (Figure 4I). At d 21, cheese treated with PC1 and PC2 had significantly lower counts $(P=0.0351$ for each comparison) compared with control cheese, with counts reaching $1.77 \pm 0.81,1.77 \pm 0.81$, and $3.42 \pm$ $0.65 \log \mathrm{cfu} / \mathrm{g}$, respectively. None of the treatments exceeded T. delbrueckii spoilage limits throughout d 21 of incubation under refrigerated conditions.

In this study, we evaluated visually the antifungal activity of protective cultures against mold in QF stored at $6 \pm 2{ }^{\circ} \mathrm{C}$, comparing mold growth on control cheese with that of cheeses with protective cultures. Examination was performed weekly over $70 \mathrm{~d}$ of incubation. Day 70 was selected as the cutoff point of the study, because negative control cheeses started showing visible growth beyond that. Tables 3 and 4 show the results of mold growth on QF surface from d 14 through 42 and from d 49 through 70 of incubation, respectively. Until d 14, mold growth was not visible on any cheeses.

For 4 mold species (Aspergillus cibarius, Trichoderma amazonicum, Penicillium citrinum, and Penicillium decumbens), no growth was observed throughout d 70 on any treatment, including the positive control. The viability of the stocks was confirmed (data not shown). Because all strains were isolated from dairy processing environments, this suggests that these 4 mold strains do not grow on $\mathrm{QF}$ under the experimental conditions.

Mucor strains tested in the study showed varying responses to protective cultures. For Muc. genevensis, all cheeses (PC1, PC2, PC3, and C) showed the "cathair" growth characteristic of Mucor spp. by d 21 of incubation. For Muc. racemosus, PC1- and PC2-treated cheeses had delayed appearance of the "cat-hair" surface at d 14 compared with $\mathrm{C}$, showing only the matte appearance indicative of partial mold growth. However,

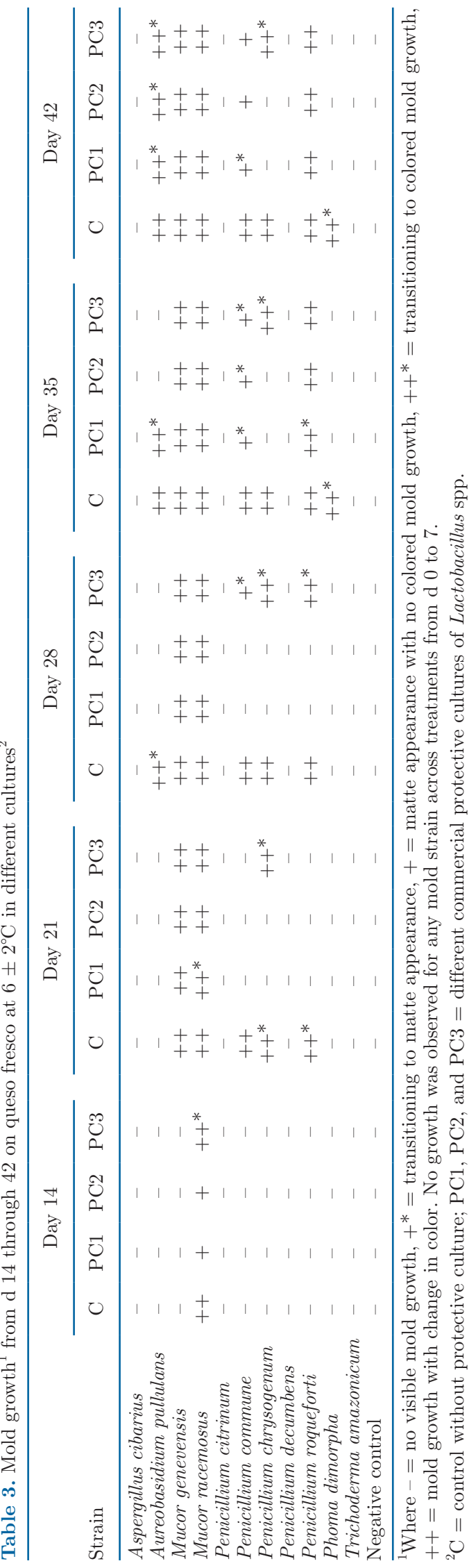




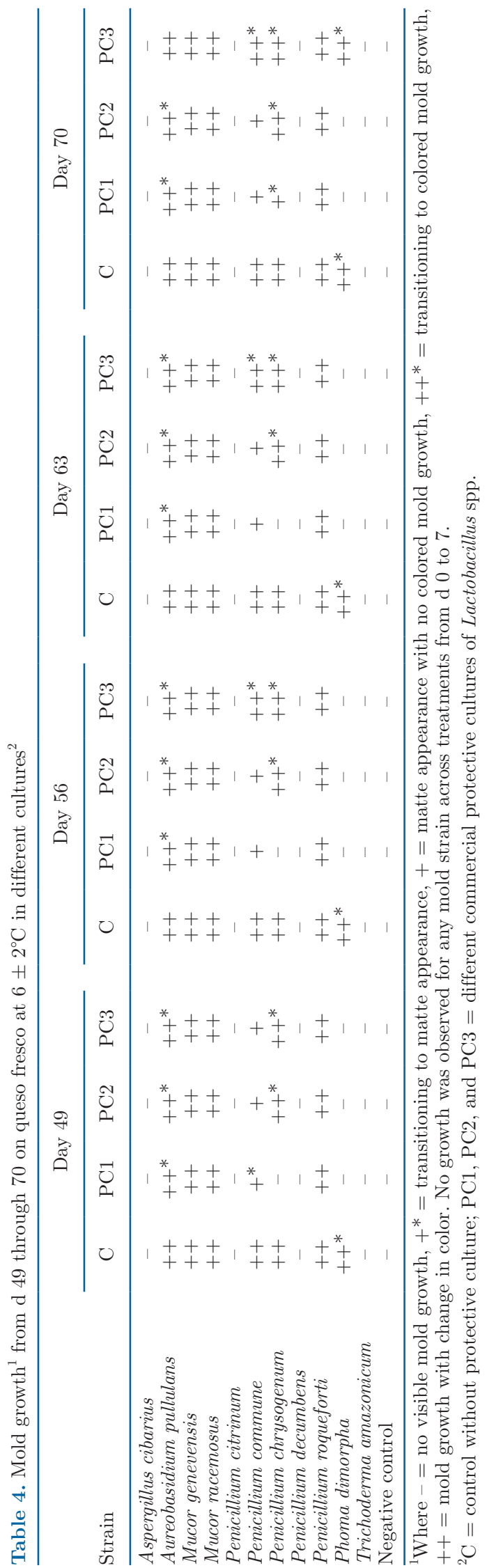

at d 21, all cheeses (PC1, PC2, PC3, and C) showed "cat-hair" growth on the cheese surface.

At d 21, visible growth of $P$. commune was only observed on control cheese with no protective culture. Only PC1- and PC2-treated cheeses continued inhibiting $P$. commune visible growth through d 28; PC3-treated cheese displayed the matte appearance indicating partial mold growth. By d 35, PC1- and PC2-treated cheeses started showing a matte surface on QF that continued throughout d 70. The PC3-treated cheese started showing green mold growth at d 56 . For P. chrysogenum, visible mold growth was observed on control cheese and on PC3-treated cheese at d 21 postinoculation. The $\mathrm{PC} 2$-treated cheese continued to inhibit visible growth of $P$. chrysogenum through d 42, and PC1 inhibited visible growth through d 63 of incubation at $6 \pm 2^{\circ} \mathrm{C}$. For $P$. roqueforti, visible mold growth was noted on PC1- and PC2-inoculated cheeses at d 35 compared with the positive control, which showed visible growth by $\mathrm{d} 21$. Visible growth was delayed by 1 wk in PC3-treated cheese compared with control, and displayed green mold growth by d 28 . Although the positive control cheese started showing visible brown growth characteristic of $P h$. dimorpha at d 35, all treatment cheeses continued to inhibit visible growth throughout d 63. At d 70, PC3-inoculated cheese started showing tan growth in a few replicates.

In summary, our results demonstrated the potential efficacy of protective cultures to protect against certain fungal spoilage in $\mathrm{QF}$ under refrigerated storage. The PC1 treatment consisting of Lactobacillus spp. strains had the broadest spectrum of efficacy across yeast and molds. Treatment with PC1 delayed spoilage caused by 5 distinct yeast strains: $C l$. lusitaniae, $D$. hansenii, D. prosopidis, Mey. guilliermondii, and $R$. mucilaginosa, and inhibited visible growth of 2 mold strains: P. chrysogenum and Ph. dimorpha. However, it is also notable that at d 21, PC1-inoculated cheese had significantly higher counts of Pichia fermentans $(P=$ 0.0018) compared with control cheese, suggesting that addition of a protective culture can have an adverse effect on yeast counts under these experimental conditions by enhancing growth of spoilage yeasts. Further studies are needed to elucidate mechanisms of action that result in enhanced growth of spoilage yeasts by the protective cultures.

Under refrigerated conditions, PC3 inhibited $\mathrm{Cl}$. lusitaniae, Mey. guilliermondii, Ph. dimorpha, and $R$. mucilaginosa on QF, whereas $\mathrm{PC} 2$ inhibited outgrowth of $C l$. lusitaniae, D. hansenii, Ph. dimorpha, and $R$. mucilaginosa.

Although PC2 inhibited D. hansenii, it did not inhibit $D$. prosopidis outgrowth, suggesting that yeast inhibition is species, not genus, selective. This was 
emphasized for molds: PC1 inhibited P. chrysogenum but only slowed $P$. roqueforti growth for 2 wk compared with the positive control.

Only 1 species of mold and 3 species of yeast were not inhibited by any of the protective cultures used in the study: Mucor genevensis, Cl. zeylanoides, Pichia fermentans, and T. delbrueckii were not visibly inhibited. This lack of inhibition suggests that successful control of these species with protective cultures may be a challenge for QF producers. This study did not cover all commercially available protective cultures, and we recommend that QF producers thoroughly evaluate the use of protective cultures against these species or other species of concern to ensure their products achieve extended shelf life.

Although few studies have investigated the use of protective cultures against individual mold and yeast strains on QF, 3 select studies show the effectiveness of protective cultures to inhibit the growth of fungal spoilage organisms in cheeses. For example, Garcha and Natt (2012) investigated the antifungal activity of Lactobacillus acidophilus, a bacteriocin-producing LAB, in vitro and in an Indian cheese variety. In vitro, $L$. acidophilus inhibited outgrowth of various mold strainsAlternaria alternata, Aspergillus flavus, Fusarium sp., and Penicillium sp.- - to different extents. Against Alternaria alternata, LAB-inoculated cheese showed significantly lower counts $(P<0.05)$ than control at d 6 of incubation at $10^{\circ} \mathrm{C}$. Our findings align with the results of that study because we demonstrated the efficacy of Lactobacillus spp. in inhibiting visible outgrowth of $P$. chrysogenum on QF through d 70 at $6 \pm 2^{\circ} \mathrm{C}$.

Furthermore, Cheong et al. (2014) screened over 800 LAB isolates for antifungal activity, of which only 12 isolates (identified as L. plantarum) demonstrated strong antifungal activity against $P$. commune using a modified overlay assay method. Cottage cheese inoculated with LAB had delayed visible mold growth to at least d 18 compared with control, which showed visible growth by d 4. Similarly, Fernandez et al. (2017) investigated the antifungal activity of over 80 commercially available LAB strains. Inhibition of $P$. commune by $L$. rhamnosus A238 alone or in combination with Bifidobacterium animalis ssp. lactis A026 in cottage cheese was reported. Our results agree with the findings of Cheong et al. (2014) and Fernandez et al. (2017); in our study, P. commune growth on QF was delayed until d 35 in cheeses treated with Lactobacillus spp. and L. rhamnosus. Last, Leyva Salas et al. (2018) demonstrated the efficacy of 2 LAB cocktails composed of Lactobacillus plantarum L244 with either Lactobacillus harbinensis L172 or L. rhamnosus CIRM-BIA1113 in slowing the growth kinetics of $P$. commune by 1 to $6 \mathrm{~d}$ and by $1 \mathrm{~d}$, respectively, on semi-hard cheese stored at $12^{\circ} \mathrm{C}$.
Overall, our data show that commercial LAB cultures vary in performance against yeast and mold strains that cause spoilage in QF. The efficacy of the 3 commercial cultures tested varied at both the genus and species level; thus, each protective culture may be ideal against a specific strain, taking into consideration food matrix factors affecting fungal growth. Manufacturers evaluating the use of protective LAB cultures should investigate efficacy against specific fungal strains of concern. Although commercial LAB cultures are marketed to manufacturers as having a natural antifungal activity, antifungal efficacy varies widely and may not be appropriately effective against fungal strains that are problematic in a given facility. Future work is needed to elucidate the mechanisms of action resulting in fungal growth inhibition, as this was beyond the scope of these studies.

\section{ACKNOWLEDGMENTS}

The authors acknowledge the support of the National Dairy Council (Rosemont, IL). The authors also acknowledge the guidance received from the Cornell Food Safety Laboratory (Ithaca, NY) in cheese making and for provision of most of the mold and yeast strains used throughout the study. The authors have not stated any conflicts of interest.

\section{REFERENCES}

Ben Said, L., H. Gaudreau, L. Dallaire, M. Tessier, and I. Fliss. 2019. Bioprotective culture: A new generation of food additives for the preservation of food quality and safety. Ind. Biotechnol. (New Rochelle N.Y.) 15:138-147. https://doi.org/10.1089/ind.2019.29175 .lbs.

Bermúdez-Aguirre, D., and G. V. Barbosa-Cánovas. 2010. Processing of soft Hispanic cheese ("queso fresco") using thermo-sonicated milk: A study of physicochemical characteristics and storage life. J. Food Sci. 75:S548-S558. https://doi.org/10.1111/j.1750-3841.2010 .01850.x.

Brown, S. R. B., E. C. Forauer, and D. J. D'Amico. 2018. Effect of modified atmosphere packaging on the growth of spoilage microorganisms and Listeria monocytogenes on fresh cheese. J. Dairy Sci. 101:7768-7779. https://doi.org/10.3168/jds.2017-14217.

Cheong, E. Y. L., A. Sandhu, J. Jayabalan, T. T. Kieu Le, N. T. Nhiep, H. T. My Ho, J. Zwielehner, N. Bansal, and M. S. Turner. 2014. Isolation of lactic acid bacteria with antifungal activity against the common cheese spoilage mould Penicillium commune and their potential as biopreservatives in cheese. Food Control 46:91-97. https://doi.org/10.1016/j.foodcont.2014.05.011.

Crowley, S., J. Mahony, and D. van Sinderen. 2013. Current perspectives on antifungal lactic acid bacteria as natural bio-preservatives. Trends Food Sci. Technol. 33:93-109. https://doi.org/10.1016/j .tifs.2013.07.004.

Fernandez, B., A. Vimont, É. Desfossés-Foucault, M. Daga, G. Arora, and I. Fliss. 2017. Antifungal activity of lactic and propionic acid bacteria and their potential as protective culture in cottage cheese. Food Control 78:350-356. https://doi.org/10.1016/j.foodcont.2017 .03 .007 .

Galvez, A., M. J. Grande Burgos, R. Lucas López, and R. Pérez Pulido. 2014. Food Biopreservation. SpringerBriefs in Food, Health, and Nutrition. Springer New York, New York, NY. 
Garcha, S., and N. K. Natt. 2012. In situ control of food spoilage fungus using Lactobacillus acidophilus NCDC 291. J. Food Sci. Technol. 49:643-648. https://doi.org/10.1007/s13197-011-0482-1.

Garnier, L., F. Valence, and J. Mounier. 2017. Diversity and control of spoilage fungi in dairy products: An update. Microorganisms 5:42. https://doi.org/10.3390/microorganisms5030042.

Guo, L., D. L. Van Hekken, P. M. Tomasula, J. Shieh, and M. H. Tunick. 2011. Effect of salt on the chemical, functional, and rheological properties of Queso Fresco during storage. Int. Dairy J. 21:352-357. https://doi.org/10.1016/j.idairyj.2010.12.009.

Lawton, M. R., K. G. Jencarelli, S. M. Kozak, and S. D. Alcaine. 2020. Short communication: Evaluation of commercial meat cultures to inhibit Listeria monocytogenes in a fresh cheese laboratory model. J. Dairy Sci. https://doi.org/10.3168/jds.2019-17203.

Leggett, L. N., P. M. Tomasula, D. L. van Hekken, A. C. S. Porto-Fett, B. Shoyer, J. A. Renye, J. B. Luchansky, and N. Farkye. 2012. Effect of storage at 4 and $10^{\circ} \mathrm{C}$ on the growth of Listeria monocytogenes in and on queso fresco. J. Food Saf. 32:236-245. https:// doi.org/10.1111/j.1745-4565.2012.00373.x.

Leyva Salas, M., A. Thierry, M. Lemaître, G. Garric, M. Harel-Oger, M. Chatel, S. Lê, J. Mounier, F. Valence, and E. Coton. 2018. Antifungal activity of lactic acid bacteria combinations in dairy mimicking models and their potential as bioprotective cultures in pilot scale applications. Front. Microbiol. 9:1787. https://doi.org/ 10.3389/fmicb.2018.01787.

Pitt, J. I., and A. D. Hocking. 2009. Fungi and Food Spoilage. 3rd ed. Springer, Dordrecht, the Netherlands.
Stiles, M. E. 1996. Biopreservation by lactic acid bacteria. Antonie van Leeuwenhoek 70:331-345. https://doi.org/10.1007/BF00395940.

Torres-Llanez, M. J., B. Vallejo-Cordoba, M. E. Díaz-Cinco, M. A. Mazorra-Manzano, and A. F. González-Córdova. 2006. Characterization of the natural microflora of artisanal Mexican Fresco cheese. Food Control 17:683-690. https://doi.org/10.1016/j foodcont.2005.04.004.

United State Census Bureau. 2018. Hispanic Population to Reach 111 Million by 2060. Accessed July 20, 2019. https://www.census.gov/ library/visualizations/2018/comm/hispanic-projected-pop.html.

Van Hekken, D. L., M. H. Tunick, J. A. Renye Jr., and P. M. Tomasula. 2017. Characterization of starter-free Queso Fresco made with sodium-potassium salt blends over 12 weeks of $4^{\circ} \mathrm{C}$ storage. J. Dairy Sci. 100:5153-5166. https://doi.org/10.3168/jds.2016-12081.

Zantar, S., F. Yedri, R. Mrabet, A. Laglaoui, M. Bakkali, and M. H. Zerrouk. 2014. Effect of Thymus vulgaris and Origanum compactum essential oils on the shelf life of fresh goat cheese. J. Essent. Oil Res. 26:76-84. https://doi.org/10.1080/10412905.2013.871673.

\section{ORCIDS}

Ghadeer M. Makki ๑ https://orcid.org/0000-0003-0373-1266

Sarah M. Kozak @ https://orcid.org/0000-0003-2538-1463

Katharine G. Jencarelli @ https://orcid.org/0000-0002-9439-7509

Samuel D. Alcaine @ https://orcid.org/0000-0001-7953-9145 